Proceedings of the International Conference on Oxide Materials for Electronic Engineering, May 29-June 2, 2017, Lviv

\title{
Highly Nonlinear Multi-Material Chalcogenide Spiral Photonic Crystal Fiber for Supercontinuum Generation
}

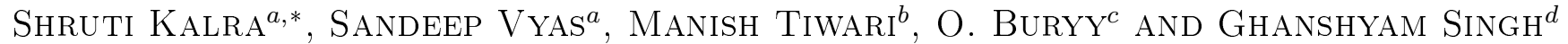 \\ ${ }^{a}$ Dept. of ECE, Jaipur Engineering College \& Research Center, Jaipur, India \\ ${ }^{b}$ Dept. of ECE, Manipal University, Jaipur, India \\ ${ }^{c}$ Inst. of Tele. Radioelectronics and Electronic Eng., Lviv Polytechnic National University, Ukraine \\ ${ }^{d}$ Dept. of ECE, Malaviya National Institute of Technology, Jaipur, India
}

\begin{abstract}
In this paper, we have numerically simulated a highly nonlinear multi-material chalcogenide photonic crystal fiber in order to investigate both the linear and the nonlinear parameters for generating a supercontinuum. The photonic crystal fiber is designed with borosilicate and $\mathrm{As}_{2} \mathrm{~S}_{3}$ glass, where borosilicate is doped in a spiral shape in the cladding region of the fiber. The designed fiber was carefully anlaysed for the parameters, effective refractive index, effective mode area, dispersion and nonlinearity coefficient. The estimated parameters are found satisfactory and suitable for further simulation and investigation of the design for broadband supercontinuum generation.
\end{abstract}

DOI: 10.12693 /APhysPolA.133.1000

PACS/topics: 42.70.Mp, 42.81.Pa, 78.20.Ci

\section{Introduction}

Photonic crystal fibers have attracted the attention of researchers since their inception due to their higher design flexibility and their enormous applications in the fields of communication, sensing, and biomedical science [1]. Photonic crystal fibers commonly known as microstructured optical fibers and holey fibers are a special class of photonic crystals, with a core surrounded by a microstructure of air-holes arrangement in a lattice forming the cladding [2].

The geometry of the photonic crystal fibers offers a greater degree of freedom to tailor its optical properties. The advancement in fabrication technology offers greater possibilities to fabricate the photonic crystal fibers with different materials other than silica [3].

Together with the flexibility to tailor the design and geometry along with a variety of material choices for fabrication, the photonic crystal fibers have not only attracted the interest of researchers but have also emerged as one of the most promising candidates for broadband supercontinuum generation [4].

Supercontinuum can be simply defined as a process resulting in extreme pulse broadening as it travels in a nonlinear medium.It can be best explained as a $\hat{A}$ nonlinear phenomenon where an intense ultra-short (narrow) laser pulse undergoes spectral broadening during its propagation in a nonlinear medium, resulting in a super-wide continuous optical spectrum. The parameters that greatly affect the generation of the supercontinuum include chromatic dispersion, peak power of the pulse, pump wavelength, pulse duration and length of the

*corresponding author; e-mail: 2013rec9033@mnit.ac.in nonlinear fiber [5]. The numerous possible applications of supercontinuum in optical coherence tomography, microscopy and spectroscopy, characterization of optical devices, multiple carrier wave generation in wavelength division multiplexing and frequency metrology and various other applications has increased their demand across the globe [6].

In the last few years photonic crystal fibers have emerged as a promising candidate for broadband supercontinuum generation in visible and infra-red region for various applications. Many materials like lead-silicate, tellurite, bismuth-oxide and chalcogenide glass offering suitable nonlinearity have been explored to fabricate photonic crystal fibers which can be engineered to generate supercontinuum for the desired range [7].

The use of nonsilica materials offers high Kerr nonlinear coefficients, and unique the characteristics of photonic crystal fibers offers enhanced flexibility to tailor parameters like effective area, dispersion and nonlinear coefficient to obtain supercontinuum generation in the desired range [3].

In this paper we present a multi-material chalcogenide glass based photonic crystal fiber designed with $\mathrm{As}_{2} \mathrm{~S}_{3}$ and borosilicate. In order to analyze its possibility to be employed for supercontinuum generation, we have numerically investigated the following parameters: effective refractive index, effective area, dispersion and nonlinear coefficient for the range of wavelength spanning from 1 to $8 \mu \mathrm{m}$.

\section{Proposed PCF design}

Based on our research work, we propose a new fiber design by replacing the air-hole in cladding region with a high refractive index material. The photonic crystal fiber consists of arsenic sulfide $\left(\mathrm{As}_{2} \mathrm{~S}_{3}\right)$ as the background material that has a hexagonal geometry with four 
rings. The effective refractive index is tailored by replacing the air-holes of the inner ring and cladding region with borosilicate. The air-holes of the cladding region are replaced in spiral pattern as depicted in Fig. 1 to tailor dispersion, effective area and nonlinearity coefficient to generate supercontinuum. The rods of borosilicate are represented with red color and air-holes are represented in black color to clearly distinguish the doped air-holes in the design. The diameter of air-hole $(d)$ and borosilicate and pitch of fiber $(\Lambda)$ are kept uniform throughout.

The numerical investigation was carried out with a tailored design with $d=1.325 \mu \mathrm{m}$ and $\Lambda=2.5 \mu \mathrm{m}$.

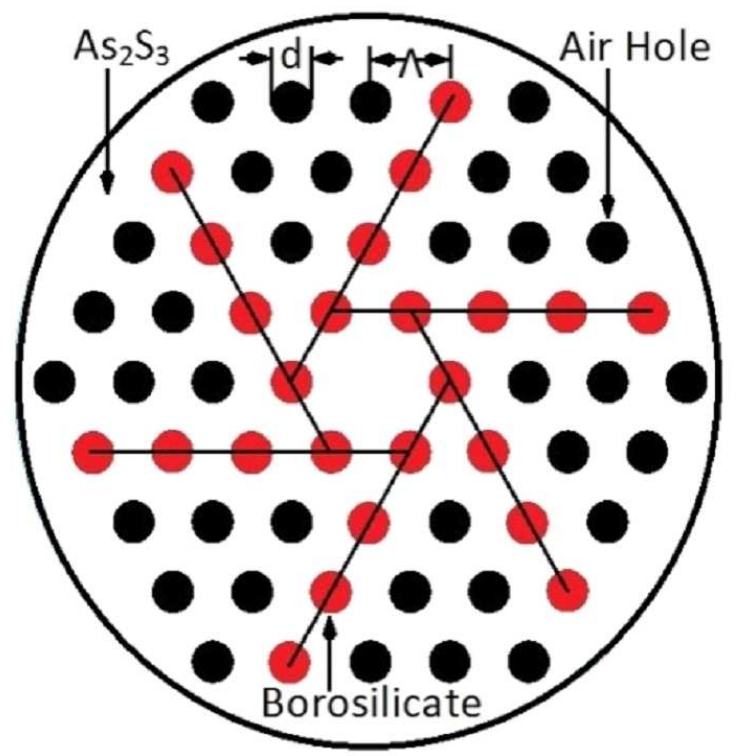

Fig. 1. Schematic design of the proposed photonic crystal fiber.

\section{Method of analysis and results}

In order to numerically investigate the characteristics of the designed fiber we have simulated the design for the wavelength varying from 1 to $8 \mu \mathrm{m}$. In our research work we mainly focused on the characteristics which play a vital role in the generation of supercontinuum generation like effective refractive index, effective area, dispersion, and nonlinear coefficient.

We can estimate the wavelength related refractive index of $\mathrm{As}_{2} \mathrm{~S}_{3}$ glass and borosilicate material by the following Sellmeier Eqs. (1) and (2), respectively, as [8]:

$$
\begin{gathered}
n^{2}(\lambda)=1+\frac{A_{1} \lambda^{2}}{\lambda^{2}-\lambda_{1}^{2}}+\frac{A_{2} \lambda^{2}}{\lambda^{2}-\lambda_{2}^{2}} \\
+\frac{A_{3} \lambda^{2}}{\lambda^{2}-\lambda_{3}^{2}}+\frac{A_{4} \lambda^{2}}{\lambda^{2}-\lambda_{4}^{2}}+\frac{A_{5} \lambda^{2}}{\lambda^{2}-\lambda_{5}^{2}},
\end{gathered}
$$

where the coefficients have the values as mentioned below: $A_{1}=1.898367, A_{2}=1.922297, A_{3}=0.87651, A_{4}=$ $0.11887, A_{5}=0.95699, \lambda_{1}=0.15 \mu \mathrm{m}, \lambda_{2}=0.25 \mu \mathrm{m}$, $\lambda_{3}=0.35 \mu \mathrm{m}, \lambda_{4}=0.45 \mu \mathrm{m}, \lambda_{5}=27.3861 \mu \mathrm{m}$, and

$$
n^{2}(\lambda)=1+\frac{B_{1} \lambda^{2}}{\lambda^{2}-\lambda_{1}^{2}}+\frac{B_{2} \lambda^{2}}{\lambda^{2}-\lambda_{2}^{2}}+\frac{B_{3} \lambda^{2}}{\lambda^{2}-\lambda_{3}^{2}},
$$

where $B_{1}=0.967, B_{2}=0.00511, B_{3}=0.34938, \lambda_{1}^{2}=$ $0.020452 \mu \mathrm{m}, \lambda_{2}^{2}=107.9261 \mu \mathrm{m}, \lambda_{3}^{2}=0.000233 \mu \mathrm{m}$.

The chromatic dispersion $\left(D_{c}\right)$ results due to both waveguide dispersion $\left(D_{W}\right)$ and material dispersion $\left(D_{M}\right)$ and can be expressed as $D_{c}(\lambda)=D_{M}(\lambda)+D_{W}(\lambda)$. Chromatic dispersion $D_{c}(\lambda)$ can be calculated using the following relation:

$$
D_{c}(\lambda)=-\frac{\lambda}{c} \frac{\mathrm{d}^{2} n_{e f f}}{\mathrm{~d} n^{2}} .
$$

In the given relation $c$ is the speed of light in vacuum, $\lambda$ is the wavelength of operation, and $n_{\text {eff } f}$ is the effective index (real part) which can be defined as $n_{\text {eff }}=\lambda \beta / 2 \pi$ where $\beta$ is the propagation constant [5]. As the expression depicts that dispersion is related to $n_{\text {eff }}$ the effective index (real part) we have simulated our design to calculate the effective index and used the values obtained to estimate the dispersion. Figure 2 depicts the plot of both effective refractive index and dispersion.

With the careful selection of the pitch $(\Lambda)$ and air-hole diameter $d$, the flat dispersion profile for the range 1.8 to $8 \mu \mathrm{m}$ was obtained with two zero dispersion wavelength (ZDW) at 2.73 and $4.57 \mu \mathrm{m}$. A flat and low dispersion profile helps enhancing the supercontinuum generation [2].

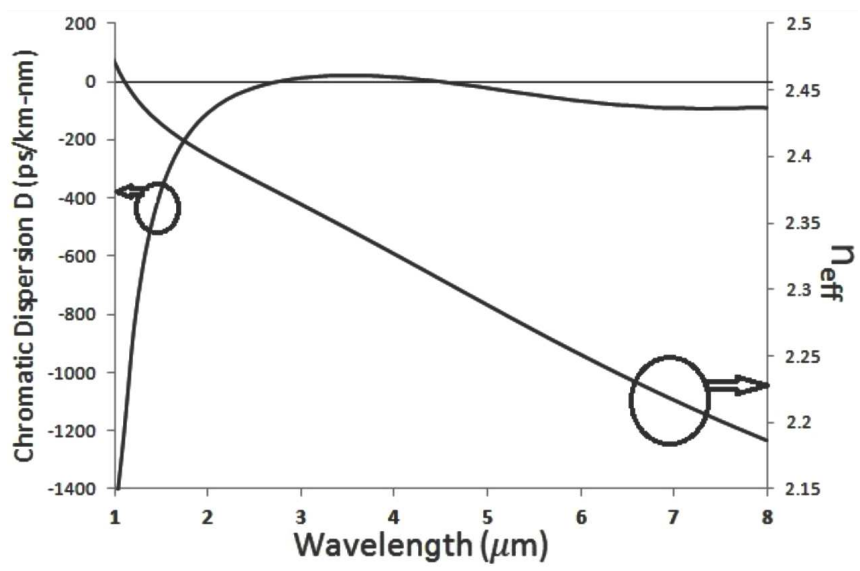

Fig. 2. The dispersion and effective refractive index plot of PCF.

The effective area of the propagating mode $\left(A_{e f f}\right)$ of the photonic crystal fiber can be determined with the given relation in Eq. 4 [5] and can also be estimated with numerical simulation.

$$
A_{e f f}=\frac{\left(\iint_{-\infty}^{\infty}|E|^{2} \mathrm{~d} x \mathrm{~d} y\right)^{2}}{\iint_{-\infty}^{\infty}|E|^{4} \mathrm{~d} x \mathrm{~d} y} .
$$

The nonlinear coefficient $(\gamma)$ can be evaluated by the following equation [5]: 


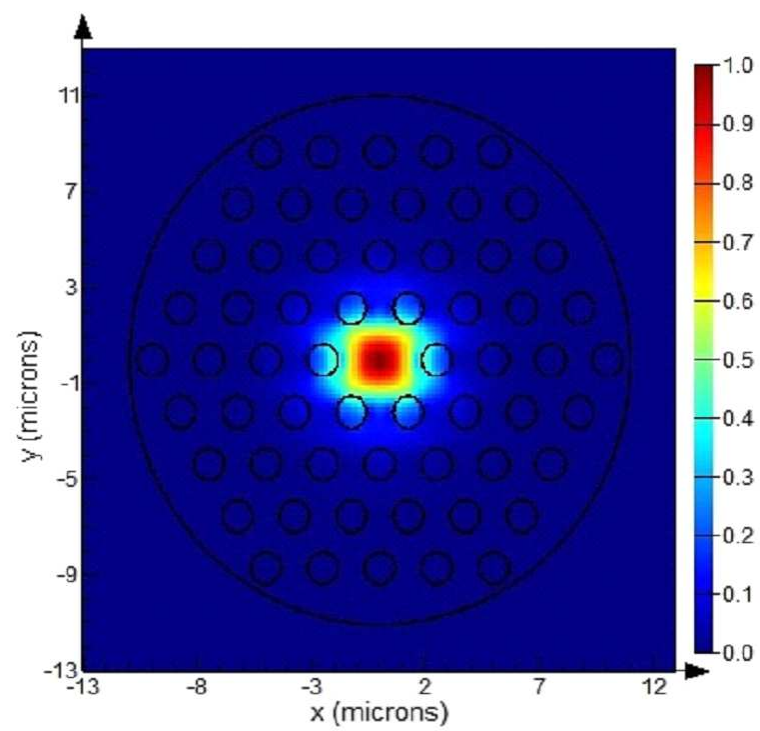

Fig. 3. The fundamental mode in the PCF.

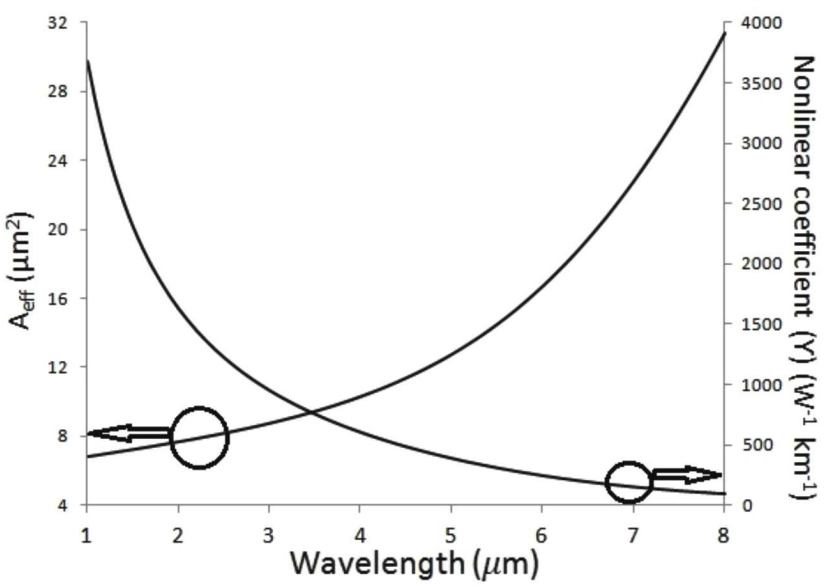

Fig. 4. The effective area and the nonlinear coefficient of the PCF.

$$
\gamma=\frac{2 \pi n_{2}}{\lambda A_{\text {eff }}},
$$

where $n_{2}$ is the refractive index of nonlinear material, $\lambda$ is pumped wavelength and $A_{e f f}$ is the effective area of the fundamental mode as shown in Fig. 3. The plot of effective area and nonlinear coefficients against the wavelength is represented in Fig. 4. Here, the nonlinear refractive index of $\mathrm{As}_{2} \mathrm{~S}_{3}$ based chalcogenide glass is $n_{2}=4 \times 10^{-18} \mathrm{~m}^{2} / \mathrm{W}$ at $2.8 \mu \mathrm{m} \mathrm{[8]}$. The plot can be used to estimate the values of both effective area and nonlinear coefficient at the desired wavelength of operation.

\section{Conclusions}

In the paper presented here a multi-material photonic crystal fiber with hexagonal lattice arrangement of airholes is numerically investigated for its characteristics like effective refractive index, effective area, nonlinear coefficients and dispersion. The range for flat dispersion of the proposed photonic crystal fiber design extends from from 1.8 to $8 \mu \mathrm{m}$.

The nonlinear coefficient $(\gamma)$, effective area $\left(A_{\text {eff }}\right)$ and dispersion at the wavelength $\lambda=2.8 \mu \mathrm{m}$ as estimated are $1052 \mathrm{~W}^{-1} \mathrm{~km}^{-1}, 8.53242 \mu \mathrm{m}^{2}$ and $4.903392 \mathrm{ps} / \mathrm{km} / \mathrm{nm}$, respectively, for the designed photonic crystal fiber. The results of the investigation are satisfactory and suitable for further investigation of the design for broadband supercontinuum generation.

\section{Acknowledgments}

The work is partially supported in the frames of the India-Ukraine intergovernmental science\&technology cooperation programme between the MNIT Jaipur (India) and the Lviv Polytechnic National University, Lviv (Ukraine) and by the Ukrainian Ministry of Education and Science (project DB/MEZHA no. 0118U000273).

\section{References}

[1] J.M. Dudley, G. Gentry, S. Coen, Rev. Mod. Phys. 78, 1135 (2006).

[2] S. Vyas, T. Tanabe, M. Tiwari, G. Singh, Chin. Opt. Lett. 14, 123201 (2016).

[3] J.M. Dudley, J.R. Taylor, Supercontinuum Generation in Optical Fibers, Cambridge University Press, 2010.

[4] S. Vyas, T. Tanabe, G. Singh, M. Tiwari, in: Proc. Int. Conf. on Computational Techniques in Information and Communication Technologies, ICCTICT, New Delhi (India), 2016, IEEE, p. $607 .$.

[5] G.P. Agarwal, Nonlinear Fiber Optics, 4th ed., Academic, 2007.

[6] S. Vyas, T. Tanabe, M. Tiwari, G. Singh, Ukr. J. Phys. Opt. 17, 132 (2016).

[7] S. Vyas, T. Tanabe, M. Tiwari, G. Singh, IEEE Int. Conf. Advances in Computing, Communications and Informatics, ICACCI, Jaipur (India), 2016, IEEE, p. 2547; S. Vyas, M. Tiwari, T. Tanabe, G. Singh, in: Proc. International Conference on Recent Cognizance in Wireless Communication \&5 Image Processing, India, Springer 2016, p.409.

[8] P.S. Maji, P.R. Chaudhuri, J. Opt. Soc. Am. 54, 4042 (2015). 